\title{
A simple algorithm for computing projection onto intersection of finite level sets
}

\author{
Songnian $\mathrm{He}^{*}$, Ziyi Zhao and Biao Luo
}

"Correspondence:

songnianhe@163.com

College of Science, Civil Aviation

University of China, Tianjin, 300300,

China

\begin{abstract}
We consider the problem of computing the projection $P_{C} u$, where $u$ is chosen in a real Hilbert space $H$ arbitrarily and the closed convex subset $C$ of $H$ is the intersection of finite level sets of convex functions given as follows:

$C=\bigcap_{i=1}^{m} C^{i} \triangleq \bigcap_{i=1}^{m}\left\{x \in H: c_{i}(x) \leq 0\right\}$, where $m$ is a positive integer and $c_{i}: H \rightarrow \mathbb{R}$ is a convex function for $i=1, \ldots, m$. A relaxed Halpern-type algorithm is proposed for computing the projection $P_{C} u$ in this paper, which is defined by $x^{n+1}=\lambda_{n} u+\left(1-\lambda_{n}\right) P_{C_{n}^{m}} \ldots P_{C_{n}^{2}} P_{C_{n}^{1}} x^{n}, n \geq 0$, where the initial guess $x^{0} \in H$ is chosen arbitrarily, the sequence $\left(\lambda_{n}\right)$ is in $(0,1)$ and $\left(C_{n}^{i}\right)$ is a sequence of half-spaces containing $C^{i}$ for $i=1, \ldots, m$. Since calculations of the projections onto half-spaces $C_{n}^{i}$ $(i=1, \ldots, m ; n=1,2, \ldots)$ are easy in practice, this algorithm is quite implementable. Strong convergence of our algorithm is proved under some ordinary conditions. Some numerical experiments are provided which show advantages of our algorithm. MSC: $58 \mathrm{E} 35 ; 47 \mathrm{H} 09 ; 65 \mathrm{~J} 15$
\end{abstract}

Keywords: projection; Hilbert space; half-space; strong convergence

\section{Introduction}

Let $H$ be a real Hilbert space with the inner product $\langle\cdot, \cdot\rangle$ and the norm $\|\cdot\|$ and let $C$ be a nonempty closed convex subset of $H$. The projection $P_{C}$ from $H$ onto $C$ is defined by

$$
P_{C} x:=\arg \min _{y \in C}\|x-y\|^{2}, \quad x \in H .
$$

It is well known that $P_{C}$ is characterized by the inequality (for $x \in H$ )

$$
\left\langle x-P_{C} x, y-P_{C} x\right\rangle \leq 0, \quad \forall y \in C .
$$

The projection operator has a variety of specific applications in different areas, such as the fixed point problem, the convex optimization problem, the variational inequality [1], the split feasibility problem [2-6], and many other applied fields. But the projection onto a general closed convex subset has no explicit expression (unless $C$ is a closed ball or halfspace and so on), so the computation of a projection is generally difficult. We know that the method of alternating projections (MAP) (also known as successive orthogonal projections (SOP)) has been thoroughly studied. The simplest and earliest convergence results concerning alternating projections between two sets were discovered by von Neumann

@2014 He et al.; licensee Springer. This is an Open Access article distributed under the terms of the Creative Commons Attribution License (http://creativecommons.org/licenses/by/2.0), which permits unrestricted use, distribution, and reproduction in any medium, provided the original work is properly cited. 
[7], Bregman [8] and Gubin et al. [9]. Now we briefly list some results of MAP. In 1933, von Neumann [7] proved that the sequence $\left(x^{n}\right)$ generated by the scheme:

$$
x^{n}=\left(P_{C^{1}} P_{C^{2}}\right)^{n} u, \quad n \geq 1,
$$

converges in norm to $P_{C^{1} \cap C^{2}} u$ when $C^{1}$ and $C^{2}$ are two closed subspaces of $H$. In 1965, Bregman [8] showed that the iterates generated by (1.2) converge weakly to $P_{C^{1} \cap C^{2}} u$ for any pair of closed convex subsets $C^{1}$ and $C^{2}$. Gubin et al. [9] proved that the iterates will converge linearly to $P_{C^{1} \cap C^{2}} u$ if $C^{1}$ and $C^{2}$ are 'boundedly regular'. Actually, they proved this result for alternating projections between any finite collection of closed convex sets. Strong convergence also holds when the sets are symmetric [10, Theorem 2.2; 11, Corollary 2.6]. However, in 2004, Hundal [12] proved that the sequence of iterates generated by (1.2) does not always converge in norm to $P_{C^{1} \cap C^{2}} u$ by providing an explicit counterexample.

Since the computation of a projection onto a closed convex subset is generally difficult, to overcome this difficulty, Fukushima [13] suggested a way to calculate the projection onto a level set of a convex function by computing a sequence of projections onto halfspaces containing the original level set. This idea is followed by Yang [3] and Lopez et al. [14], respectively, who introduced the relaxed CQ algorithms for solving the split feasibility problem in the setting of finite-dimensional and infinite-dimensional Hilbert spaces, respectively. The idea is also used by Gibali et al. [11] and He et al. [15] for solving variational inequalities in a Hilbert space.

The main purpose of this paper is to consider the problem of computing the projection $P_{C} u$, where $u$ is chosen in $H$ arbitrarily and the closed convex subset $C$ is of the particular structure, i.e., the intersection of finite level sets of convex functions given as follows:

$$
C=\bigcap_{i=1}^{m} C^{i} \triangleq \bigcap_{i=1}^{m}\left\{x \in H: c_{i}(x) \leq 0\right\},
$$

where $m$ is a positive integer and $c_{i}: H \rightarrow \mathbb{R}$ is a convex function for $i=1, \ldots, m$.

A relaxed Halpern-type algorithm proposed for computing the projection $P_{C} u$ in this paper is defined by

$$
x^{n+1}=\lambda_{n} u+\left(1-\lambda_{n}\right) P_{C_{n}^{m}} \cdots P_{C_{n}^{2}} P_{C_{n}^{1}} x^{n}, \quad n \geq 0,
$$

where the initial guess $x^{0} \in H$ is chosen arbitrarily, the sequence $\left(\lambda_{n}\right)$ is in $(0,1)$ and $\left(C_{n}^{i}\right)$ is a sequence of half-spaces containing $C^{i}$ for $i=1, \ldots, m$ (the specific structure of the half-spaces $C_{n}^{i}$ will be described in Section 3). Since calculations of the projections onto half-spaces $C_{n}^{i}(i=1, \ldots, m ; n=1,2, \ldots)$ are easy in practice, this algorithm is quite implementable. Moreover, strong convergence of our algorithm can be proved under some ordinary conditions.

The rest of this paper is organized as follows. Some useful lemmas are given in Section 2. In Section 3, the strong convergence of our algorithm is proved. Some numerical experiments are given in Section 4 which show advantages of our algorithm. 


\section{Preliminaries}

Throughout the rest of this paper, we denote by $H$ a real Hilbert space. We will use the notations:

- $\rightarrow$ denotes strong convergence.

- $\rightarrow$ denotes weak convergence.

- $\omega_{w}\left(x^{n}\right)=\left\{x \mid \exists\left\{x^{n_{k}}\right\} \subset\left\{x^{n}\right\}\right.$ such that $\left.x^{n_{k}} \rightarrow x\right\}$ denotes the weak $\omega$-limit set of $\left\{x^{n}\right\}$.

Recall that a mapping $T: H \rightarrow H$ is said to be nonexpansive if

$$
\|T x-T y\| \leq\|x-y\|, \quad x, y \in H .
$$

$T: H \rightarrow H$ is said to be firmly nonexpansive if, for $x, y \in H$,

$$
\|T x-T y\|^{2} \leq\|x-y\|^{2}-\|(I-T) x-(I-T) y\|^{2} .
$$

Recall some definitions which are also useful for us to prove the main results. Recall that an element $g \in H$ is said to be a subgradient of $f: H \rightarrow \mathbb{R}$ at $x$ if

$$
f(z) \geq f(x)+\langle g, z-x\rangle, \quad \forall z \in H .
$$

A function $f: H \rightarrow \mathbb{R}$ is said to be subdifferentiable at $x$, if it has at least one subgradient at $x$. The set of subgradients of $f$ at the point $x$, denoted by $\partial f(x)$, is called the subdifferential of $f$ at $x$. The last relation above is called the subdifferential inequality of $f$ at $x$. A function $f$ is called subdifferentiable, if it is subdifferentiable at all $x \in H$.

Recall that a function $f: H \rightarrow \mathbb{R}$ is said to be weakly lower semi-continuous (w-lsc) at $x$ if $x^{n} \rightarrow x$ implies

$$
f(x) \leq \liminf _{n \rightarrow \infty} f\left(x^{n}\right)
$$

Lemma 2.1 For all $x, y \in H$, we have the relation

$$
\|x+y\|^{2} \leq\|x\|^{2}+2\langle y, x+y\rangle .
$$

This inequality is trivial but in common use.

The following lemma is the key to the proofs of strong convergence of our algorithms. In fact, it can be used as a new fundamental tool for solving some nonlinear problems, particularly, some problems related to projection operator.

Lemma 2.2 [15] Assume $\left(s_{n}\right)$ is a sequence of nonnegative real numbers such that

$$
\begin{aligned}
& s_{n+1} \leq\left(1-\gamma_{n}\right) s_{n}+\gamma_{n} \delta_{n}, \quad n \geq 0, \\
& s_{n+1} \leq s_{n}-\eta_{n}+\alpha_{n}, \quad n \geq 0,
\end{aligned}
$$

where $\left(\gamma_{n}\right)$ is a sequence in $(0,1),\left(\eta_{n}\right)$ is a sequence of nonnegative real numbers and $\left(\delta_{n}\right)$ and $\left(\alpha_{n}\right)$ are two sequences in $\mathbb{R}$ such that

(i) $\sum_{n=0}^{\infty} \gamma_{n}=\infty$, 
(ii) $\lim _{n \rightarrow \infty} \alpha_{n}=0$,

(iii) $\lim _{k \rightarrow \infty} \eta_{n_{k}}=0$ implies $\lim \sup _{k \rightarrow \infty} \delta_{n_{k}} \leq 0$ for any subsequence $\left(n_{k}\right) \subset(n)$.

Then $\lim _{n \rightarrow \infty} s_{n}=0$.

\section{Iterative algorithms}

In this section, we precisely introduce algorithm (1.3) and analyze its strong convergence. For the sake of simplicity, it suffices to consider the case $m=2$ without loss of the generality, that is, $C=C^{1} \cap C^{2}$, where

$$
\begin{aligned}
& C^{1}=\left\{x \in H: c_{1}(x) \leq 0\right\}, \\
& C^{2}=\left\{x \in H: c_{2}(x) \leq 0\right\},
\end{aligned}
$$

and $c_{1}: H \rightarrow \mathbb{R}$ and $c_{2}: H \rightarrow \mathbb{R}$ are two convex functions. We always assume that $c_{1}$ and $c_{2}$ are subdifferentiable on $H, \partial c_{1}$ and $\partial c_{2}$ are bounded operators (i.e. bounded on bounded sets). It is worth noting that every convex function defined on a finite-dimensional Hilbert space is subdifferentiable and its subdifferential operator is a bounded operator (see [16]). Suppose the $n$th iteraction $x^{n}$ has been constructed, by using the subdifferential inequality, we construct the two half-spaces as follows:

$$
\begin{aligned}
& C_{n}^{1}=\left\{x \in H: c_{1}\left(x^{n}\right) \leq\left\langle\xi_{n}^{1}, x^{n}-x\right\rangle\right\}, \\
& C_{n}^{2}=\left\{x \in H: c_{2}\left(P_{C_{n}^{1}} x^{n}\right) \leq\left\langle\xi_{n}^{2}, P_{C_{n}^{1}} x^{n}-x\right\rangle\right\},
\end{aligned}
$$

where $\xi_{n}^{1} \in \partial c_{1}\left(x^{n}\right), \xi_{n}^{2} \in \partial c_{2}\left(P_{C_{n}^{1}} x^{n}\right)$. By the subdifferential inequality, it is easy to see that

$$
\begin{aligned}
& C_{n}^{1} \supset C^{1}=\left\{x \in H: c_{1}(x) \leq 0\right\}, \\
& C_{n}^{2} \supset C^{2}=\left\{x \in H: c_{2}(x) \leq 0\right\} .
\end{aligned}
$$

Algorithm 3.1 For a given $u \in H$, take an initial guess $x^{0} \in H$ arbitrarily and construct the sequence $\left(x^{n}\right)$ via the formula

$$
x^{n+1}=\lambda_{n} u+\left(1-\lambda_{n}\right) P_{C_{n}^{2}} P_{C_{n}^{1}} x^{n}, \quad n \geq 0,
$$

where $C_{n}^{1}$ and $C_{n}^{2}$ are given by (3.2) and the sequence $\left(\lambda_{n}\right)$ is in $(0,1)$.

Theorem 3.2 Assume that $\lambda_{n} \rightarrow 0(n \rightarrow \infty)$ and $\sum_{n=1}^{+\infty} \lambda_{n}=+\infty$. Then the sequence $\left(x^{n}\right)$ generated by Algorithm 3.1 converges strongly to the point $P_{C^{1} \cap C^{2}} u$.

Proof Firstly, we verify that $\left(x^{n}\right)$ is bounded. Setting $x^{*}=P_{C^{1} \cap C^{2}} u$, since $C^{1} \cap C^{2} \subset C_{n}^{1} \cap C_{n}^{2}$, we obtain from (3.4) and Lemma 2.1 that

$$
\begin{aligned}
\left\|x^{n+1}-x^{*}\right\|^{2}= & \left\|\lambda_{n} u+\left(1-\lambda_{n}\right) P_{C_{n}^{2}} P_{C_{n}^{1}} x^{n}-x^{*}\right\|^{2} \\
= & \left\|\lambda_{n}\left(u-x^{*}\right)+\left(1-\lambda_{n}\right)\left(P_{C_{n}^{2}} P_{C_{n}^{1}} x^{n}-P_{C_{n}^{2}} P_{C_{n}^{1}} x^{*}\right)\right\|^{2} \\
\leq & \left(1-\lambda_{n}\right)\left\|P_{C_{n}^{2}} P_{C_{n}^{1}} x^{n}-P_{C_{n}^{2}} P_{C_{n}^{1}} x^{*}\right\|^{2} \\
& +2 \lambda_{n}\left(u-x^{*}, x^{n+1}-x^{*}\right\rangle
\end{aligned}
$$




$$
\begin{aligned}
\leq & \left(1-\lambda_{n}\right)\left\|x^{n}-x^{*}\right\|^{2}+2 \lambda_{n}\left\|u-x^{*}\right\| \cdot\left\|x^{n+1}-x^{*}\right\| \\
\leq & \left(1-\lambda_{n}\right)\left\|x^{n}-x^{*}\right\|^{2}+\frac{1}{4} \lambda_{n}\left\|x^{n+1}-x^{*}\right\|^{2} \\
& +4 \lambda_{n}\left\|u-x^{*}\right\|^{2}
\end{aligned}
$$

consequently

$$
\left\|x^{n+1}-x^{*}\right\|^{2} \leq \frac{1-\lambda_{n}}{1-\frac{1}{4} \lambda_{n}}\left\|x^{n}-x^{*}\right\|^{2}+\frac{\frac{3}{4} \lambda_{n}}{1-\frac{1}{4} \lambda_{n}} \frac{16}{3}\left\|u-x^{*}\right\|^{2}
$$

it turns out that

$$
\left\|x^{n+1}-x^{*}\right\| \leq \max \left\{\left\|x^{n}-x^{*}\right\|, \frac{4 \sqrt{3}}{3}\left\|u-x^{*}\right\|\right\}
$$

inductively

$$
\left\|x^{n}-x^{*}\right\| \leq \max \left\{\left\|x^{0}-x^{*}\right\|, \frac{4 \sqrt{3}}{3}\left\|u-x^{*}\right\|\right\}
$$

which means that $\left(x^{n}\right)$ is bounded.

Secondly, we use Lemma 2.2 to prove the strong convergence of Algorithm 3.1. Since a projection is firmly nonexpansive, we obtain

$$
\begin{aligned}
& \left\|P_{C_{n}^{2}} P_{C_{n}^{1}} x^{n}-P_{C_{n}^{2}} P_{C_{n}^{1}} x^{*}\right\|^{2} \\
& \quad \leq\left\|P_{C_{n}^{1}} x^{n}-P_{C_{n}^{1}} x^{*}\right\|^{2}-\left\|P_{C_{n}^{1}} x^{n}-P_{C_{n}^{2}} P_{C_{n}^{1}} x^{n}\right\|^{2} \\
& \quad \leq\left\|x^{n}-x^{*}\right\|^{2}-\left\|x^{n}-P_{C_{n}^{1}} x^{n}\right\|^{2}-\left\|P_{C_{n}^{1}} x^{n}-P_{C_{n}^{2}} P_{C_{n}^{1}} x^{n}\right\|^{2} .
\end{aligned}
$$

Using (3.6) and the first inequality of (3.5), we have

$$
\begin{aligned}
\left\|x^{n+1}-x^{*}\right\|^{2} \leq & \left(1-\lambda_{n}\right)\left\|P_{C_{n}^{2}} P_{C_{n}^{1}} x^{n}-P_{C_{n}^{2}} P_{C_{n}^{1}} x^{*}\right\|^{2} \\
& +2 \lambda_{n}\left\langle u-x^{*}, x^{n+1}-x^{*}\right\rangle \\
\leq & \left\|x^{n}-x^{*}\right\|^{2}-\left\|x^{n}-P_{C_{n}^{1}} x^{n}\right\|^{2} \\
& -\left\|P_{C_{n}^{1}} x^{n}-P_{C_{n}^{2}} P_{C_{n}^{1}} x^{n}\right\|^{2}+M \lambda_{n},
\end{aligned}
$$

where $M$ is some positive constant such that $2\left\|u-x^{*}\right\| \cdot\left\|x^{n+1}-x^{*}\right\| \leq M$ (noting that ( $x^{n}$ ) is bounded).

Setting

$$
\begin{aligned}
& s_{n}=\left\|x^{n}-x^{*}\right\|^{2}, \quad \alpha_{n}=M \lambda_{n}, \\
& \eta_{n}=\left\|x^{n}-P_{C_{n}^{1}} x^{n}\right\|^{2}+\left\|P_{C_{n}^{1}} x^{n}-P_{C_{n}^{2}} P_{C_{n}^{1}} x^{n}\right\|^{2},
\end{aligned}
$$

then (3.7) is rewritten as follows:

$$
s_{n+1} \leq s_{n}-\eta_{n}+\alpha_{n} .
$$


From the first inequality of (3.5), we also have

$$
\left\|x^{n+1}-x^{*}\right\|^{2} \leq\left(1-\lambda_{n}\right)\left\|x^{n}-x^{*}\right\|^{2}+2 \lambda_{n}\left\langle u-x^{*}, x^{n+1}-x^{*}\right\rangle .
$$

Setting

$$
\begin{aligned}
& s_{n}=\left\|x^{n}-x^{*}\right\|^{2}, \\
& \delta_{n}=2\left\langle u-x^{*}, x^{n+1}-x^{*}\right\rangle,
\end{aligned}
$$

then (3.9) is rewritten as follows:

$$
s_{n+1} \leq\left(1-\lambda_{n}\right) s_{n}+\lambda_{n} \delta_{n}
$$

Observing that the condition $\lambda_{n} \rightarrow 0$ implies $\alpha_{n} \rightarrow 0$ and the condition $\sum_{n=1}^{+\infty} \lambda_{n}=+\infty$ holds, we assert from (3.8) and (3.10) that in order to complete the proof using Lemma 2.2, it suffices to verify that

$$
\lim _{k \rightarrow \infty} \eta_{n_{k}}=0
$$

implies

$$
\limsup _{k \rightarrow \infty} \delta_{n_{k}} \leq 0
$$

for any subsequence $\left(n_{k}\right) \subset(n)$. In fact, if $\eta_{n_{k}} \rightarrow 0$ as $k \rightarrow \infty$, then $\left\|x^{n_{k}}-P_{C_{n_{k}}^{1}} x^{n_{k}}\right\| \rightarrow 0$ and $\left\|P_{C_{n_{k}}^{1}} x^{n_{k}}-P_{C_{n_{k}}^{2}} P_{C_{n_{k}}^{1}} x^{n_{k}}\right\| \rightarrow 0$ hold. Since $\partial c_{1}$ and $\partial c_{2}$ are bounded on bounded sets, there are two positive constants $\kappa_{1}$ and $\kappa_{2}$ such that $\left\|\xi_{n_{k}}^{1}\right\| \leq \kappa_{1}$ and $\left\|\xi_{n_{k}}^{2}\right\| \leq \kappa_{2}$ for all $k \geq$ 0 (noting that $P_{C_{n_{k}}^{1}} x^{n_{k}}$ is also bounded due to the fact that $\left\|P_{C_{n_{k}}^{1}} x^{n_{k}}-x^{*}\right\|=\| P_{C_{n_{k}}^{1}} x^{n_{k}}-$ $\left.P_{C_{n_{k}}^{1}} x^{*}\|\leq\| x^{n_{k}}-x^{*} \|\right)$. From the trivial fact that $P_{C_{n_{k}}^{1}} x^{n_{k}} \in C_{n_{k}}^{1}$ and $P_{C_{n_{k}}^{2}} P_{C_{n_{k}}^{1}} x^{n_{k}} \in C_{n_{k}}^{2}$, it follows that

$$
c_{1}\left(x^{n_{k}}\right) \leq\left\langle\xi_{n_{k}}^{1}, x^{n_{k}}-P_{C_{n_{k}}^{1}} x^{n_{k}}\right\rangle \leq \kappa_{1}\left\|x^{n_{k}}-P_{C_{n_{k}}^{1}} x^{n_{k}}\right\| \rightarrow 0
$$

and

$$
\begin{aligned}
c_{2}\left(P_{C_{n_{k}}^{1}} x^{n_{k}}\right) & \leq\left\langle\xi_{n_{k}}^{2}, P_{C_{n_{k}}^{1}} x^{n_{k}}-P_{C_{n_{k}}^{2}} P_{C_{n_{k}}^{1}} x^{n_{k}}\right\rangle \\
& \leq \kappa_{2}\left\|P_{C_{n_{k}}^{1}} x^{n_{k}}-P_{C_{n_{k}}^{2}} P_{C_{n_{k}}^{1}} x^{n_{k}}\right\| \rightarrow 0 .
\end{aligned}
$$

Take $x^{\prime} \in \omega_{w}\left(x^{n_{k}}\right)$ arbitrarily and assume that $x^{n_{k}} \rightarrow x^{\prime}$ holds without loss of generality, then the w-lsc of $c_{1}$ and (3.11) imply that

$$
c_{1}\left(x^{\prime}\right) \leq \liminf _{k \rightarrow \infty} c_{1}\left(x^{n_{k}}\right) \leq 0
$$

this means that $x^{\prime} \in C^{1}$ holds. Noting that $\left\|x^{n_{k}}-P_{C_{n_{k}}^{1}} x^{n_{k}}\right\| \rightarrow 0$ implies $P_{C_{n_{k}}^{1}} x^{n_{k}} \rightarrow x^{\prime}$, this together with (3.12) and the w-lsc of $c_{2}$ leads to the fact that

$$
c_{2}\left(x^{\prime}\right) \leq \liminf _{k \rightarrow \infty} c_{2}\left(P_{C_{n_{k}}^{1}} x^{n_{k}}\right) \leq 0,
$$


which implies that $x^{\prime} \in C^{2}$. Moreover, we obtain $x^{\prime} \in C^{1} \cap C^{2}$, and hence

$$
\omega_{w}\left(x^{n_{k}}\right) \subset C^{1} \cap C^{2} .
$$

On the other hand

$$
\begin{aligned}
\left\|x^{n_{k}}-x^{n_{k}+1}\right\|= & \left\|\lambda_{n_{k}}\left(x^{n_{k}}-u\right)+\left(1-\lambda_{n_{k}}\right)\left(x^{n_{k}}-P_{C_{n_{k}}^{2}} P_{C_{n_{k}}^{1}} x^{n_{k}}\right)\right\| \\
= & \| \lambda_{n_{k}}\left(x^{n_{k}}-u\right)+\left(1-\lambda_{n_{k}}\right)\left(x^{n_{k}}-P_{C_{n_{k}}^{1}} x^{n_{k}}+P_{C_{n_{k}}^{1}} x^{n_{k}}\right. \\
& \left.-P_{C_{n_{k}}^{2}} P_{C_{n_{k}}^{1}} x^{n_{k}}\right) \| \\
\leq & \lambda_{n_{k}}\left\|x^{n_{k}}-u\right\|+\left(1-\lambda_{n_{k}}\right)\left\|x^{n_{k}}-P_{C_{n_{k}}^{1}} x^{n_{k}}\right\| \\
& +\left(1-\lambda_{n_{k}}\right)\left\|P_{C_{n_{k}}^{1}} x^{n_{k}}-P_{C_{n_{k}}^{2}} P_{C_{n_{k}}^{1}} x^{n_{k}}\right\| .
\end{aligned}
$$

Letting $k \rightarrow \infty$ yields

$$
\left\|x^{n_{k}}-x^{n_{k}+1}\right\| \rightarrow 0
$$

we can deduce from (3.13) and (1.1) that

$$
\begin{aligned}
\limsup _{k \rightarrow \infty} 2\left\langle u-x^{*}, x^{n_{k}+1}-x^{*}\right\rangle & =\limsup _{k \rightarrow \infty} 2\left\langle u-x^{*}, x^{n_{k}}-x^{*}\right\rangle \\
& =\max _{w \in \omega_{w}\left(x^{n_{k}}\right)} 2\left\langle u-x^{*}, w-x^{*}\right\rangle \\
& \leq 0,
\end{aligned}
$$

which implies that

$$
\limsup _{k \rightarrow \infty} \delta_{n_{k}} \leq 0
$$

for any subsequence $\left(n_{k}\right) \subset(n)$. From the Lemma 2.2, we get $\lim _{n \rightarrow \infty} s_{n}=0$, which means $x^{n} \rightarrow x^{*}=P_{C^{1} \cap C^{2}} u$.

Now we turn to a sketch of the general case. Let $m$ be a positive integer and let $C^{i}=\{x \in$ $\left.H: c_{i}(x) \leq 0\right\}$ be a level set of a convex function $c_{i}: H \rightarrow \mathbb{R}$ for $i=1,2, \ldots, m$. We always assume that $c_{i}$ is subdifferentiable on $H$ and $\partial c_{i}$ is a bounded operator for all $i=1,2, \ldots, m$. Suppose that the $n$th iterate $x^{n}$ has been obtained, similar to (3.2), we construct $m$ halfspaces from the subdifferential inequality as follows:

$$
\begin{aligned}
& C_{n}^{1}=\left\{x \in H: c_{1}\left(x^{n}\right) \leq\left\langle\xi_{n}^{1}, x^{n}-x\right\rangle\right\}, \\
& C_{n}^{2}=\left\{x \in H: c_{2}\left(P_{C_{n}^{1}} x^{n}\right) \leq\left\langle\xi_{n}^{2}, P_{C_{n}^{1}} x^{n}-x\right\rangle\right\}, \\
& \cdots \\
& C_{n}^{m}=\left\{x \in H: c_{m}\left(P_{C_{n}^{m-1}} \cdots P_{C_{n}^{2}} P_{C_{n}^{1}} x^{n}\right) \leq\left\langle\xi_{n}^{m}, P_{C_{n}^{m-1}} \cdots P_{C_{n}^{2}} P_{C_{n}^{1}} x^{n}-x\right\rangle\right\},
\end{aligned}
$$

where $\xi_{n}^{1} \in \partial c_{1}\left(x^{n}\right), \xi_{n}^{2} \in \partial c_{2}\left(P_{C_{n}^{1}} x^{n}\right), \ldots, \xi_{n}^{m} \in \partial c_{m}\left(P_{C_{n}^{m-1}} \cdots P_{C_{n}^{2}} P_{C_{n}^{1}} x^{n}\right)$. By the subdifferential inequality, it is easy to see that $C_{n}^{i} \supset C^{i}$ holds for all $n \geq 0$ and $i=1, \ldots, m$. 
Algorithm 3.3 For a given $u \in H$, take an initial guess $x^{0} \in H$ arbitrarily and construct the sequence $\left(x^{n}\right)$ via the formula

$$
x^{n+1}=\lambda_{n} u+\left(1-\lambda_{n}\right) P_{C_{n}^{m}} \cdots P_{C_{n}^{2}} P_{C_{n}^{1}} x^{n}, \quad n \geq 0,
$$

where $C_{n}^{1}, C_{n}^{2}, \ldots, C_{n}^{m}$ are given by $(3.15)$ and the sequence $\left(\lambda_{n}\right)$ is in $(0,1)$.

By an argument very similar to the proof of Theorem 3.2, it is not difficult to see that our result of Theorem 3.2 can be extended easily to the general case.

Theorem 3.4 Assume that $\lambda_{n} \rightarrow 0(n \rightarrow \infty)$ and $\sum_{n=1}^{+\infty} \lambda_{n}=+\infty$. Then the sequence $\left(x^{n}\right)$ generated by Algorithm 3.3 converges strongly to the point $P_{C^{1} \cap C^{2} \cap \ldots \cap C^{m}} u$.

Finally, we point out that if the computation of the projection operator $P_{C^{i}}$ is easy for all $i=1, \ldots, m$ (for example, $C^{i}$ is a closed ball or a half-space for all $i=1, \ldots, m$ ), then we have no need to adopt the relaxation technique in the algorithm designs, that is, one can use the following algorithm to compute the projection $P_{C^{1} \cap C^{2} \cap \ldots \cap C^{m}} u$ for a given point $u \in H$. Moreover, the strong convergence of this algorithm can be proved by an argument similar to the proof of Theorem 3.4 (in fact, its proof is much simpler than that of Theorem 3.4).

Algorithm 3.5 Let $u \in H$ and start an initial guess $x^{0} \in H$ arbitrarily. The sequence $\left(x^{n}\right)$ is constructed via the formula

$$
x^{n+1}=\lambda_{n} u+\left(1-\lambda_{n}\right) P_{C^{m}} \cdots P_{C^{2}} P_{C^{1}} x^{n}, \quad n \geq 0,
$$

where the sequence $\left(\lambda_{n}\right)$ is in $(0,1)$.

Theorem 3.6 Assume that $\lambda_{n} \rightarrow 0(n \rightarrow \infty)$ and $\sum_{n=1}^{+\infty} \lambda_{n}=+\infty$. Then the sequence $\left(x^{n}\right)$ generated by Algorithm 3.5 converges strongly to the point $P_{C^{1} \cap C^{2} \cap \ldots \cap C^{m}} u$.

\section{Numerical experiments}

In this section, in order to show advantages of our algorithms, we present some numerical results via implementing Algorithm 3.1 and Algorithm 3.5 for two examples, respectively, in the setting of finite-dimensional Hilbert space. The codes were written in Matlab 2013a and run on an Amd Liano APU A4-3300M Core4 CPU k43t (CPU 1.9 GHz) personal computer. In the following two examples, we always take $H=\mathbb{R}^{3}$ and $\lambda_{n}=\frac{1}{n}$ for $n \geq 1$. The $n$th step iterate is denoted by $x^{n}=\left(x_{1}^{n}, x_{2}^{n}, x_{3}^{n}\right)^{\top}$. Since we do not know the exact projection $P_{C^{1} \cap C^{2}} u$, we use $E_{n} \triangleq \frac{\left\|x^{n+1}-x^{n}\right\|}{\left\|x^{n}\right\|}$ to measure the error of the $n$th step iteration.

Example 4.1 Take $u=(5,2,1)^{\top}, x^{0}=(1,5,2)^{\top}$, and

$$
\begin{aligned}
& C^{1}=\left\{x=\left(x_{1}, x_{2}, x_{3}\right)^{\top}: \frac{x_{1}^{2}}{4}+\frac{x_{2}^{2}}{9}+\frac{x_{3}^{2}}{16}-1 \leq 0\right\}, \\
& C^{2}=\left\{x=\left(x_{1}, x_{2}, x_{3}\right)^{\top}: \frac{\left(x_{1}-1\right)^{2}}{4}+\frac{\left(x_{2}-\frac{3}{2}\right)^{2}}{9}+\frac{\left(x_{3}-2\right)^{2}}{16}-1 \leq 0\right\} .
\end{aligned}
$$

Use Algorithm 3.1 to calculate the projection $P_{C^{1} \cap C^{2}} u$. 
Table 1 Numerical results as regards Example 4.1

\begin{tabular}{rllll}
\hline $\boldsymbol{n}$ & $\boldsymbol{x}_{\mathbf{1}}^{\boldsymbol{n}}$ & $\boldsymbol{x}_{\mathbf{2}}^{\boldsymbol{n}}$ & $\boldsymbol{x}_{\mathbf{3}}^{\boldsymbol{n}}$ & $\boldsymbol{E}_{\boldsymbol{n}}$ \\
\hline 1 & 4.269110 & 1.992852 & 0.999360 & $1.33 \mathrm{E}-01$ \\
110 & 3.272412 & 1.954756 & 0.995899 & $4.00 \mathrm{E}-03$ \\
262 & 3.165479 & 1.951198 & 0.995565 & $1.82 \mathrm{E}-03$ \\
300 & 3.397080 & 1.957359 & 0.996125 & $1.32 \mathrm{E}-03$ \\
398 & 3.080705 & 1.948784 & 0.995342 & $1.28 \mathrm{E}-03$ \\
466 & 3.360174 & 1.956241 & 0.996020 & $8.78 \mathrm{E}-04$ \\
509 & 3.498434 & 1.959931 & 0.996356 & $7.15 \mathrm{E}-04$ \\
671 & 3.187363 & 1.951509 & 0.995587 & $7.01 \mathrm{E}-04$ \\
891 & 2.840956 & 1.942152 & 0.994733 & $6.77 \mathrm{E}-04$ \\
969 & 3.010468 & 1.946694 & 0.995147 & $5.53 \mathrm{E}-04$ \\
987 & 3.050742 & 1.947773 & 0.995245 & $5.27 \mathrm{E}-04$ \\
1,000 & 3.076057 & 1.948451 & 0.995307 & $5.10 \mathrm{E}-04$ \\
\hline
\end{tabular}

Table 2 Numerical results as regards Example 4.2

\begin{tabular}{rllll}
\hline $\boldsymbol{n}$ & $\boldsymbol{x}_{\mathbf{1}}^{\boldsymbol{n}}$ & $\boldsymbol{x}_{\mathbf{2}}^{\boldsymbol{n}}$ & $\mathbf{x}_{\mathbf{3}}^{\boldsymbol{n}}$ & $\boldsymbol{E}_{\boldsymbol{n}}$ \\
\hline 1 & 2.298142 & 4.596285 & 5.745356 & $1.16 \mathrm{E}-01$ \\
110 & 0.626949 & 1.253898 & 1.567372 & $9.69 \mathrm{E}-03$ \\
262 & 0.609227 & 1.218453 & 1.523067 & $3.94 \mathrm{E}-03$ \\
311 & 0.607194 & 1.214388 & 1.517985 & $3.45 \mathrm{E}-03$ \\
371 & 0.605435 & 1.210869 & 1.513586 & $2.98 \mathrm{E}-03$ \\
422 & 0.604331 & 1.208663 & 1.510829 & $2.63 \mathrm{E}-03$ \\
504 & 0.603025 & 1.206050 & 1.507562 & $2.02 \mathrm{E}-03$ \\
575 & 0.602194 & 1.204388 & 1.505485 & $1.77 \mathrm{E}-03$ \\
611 & 0.601846 & 1.203693 & 1.504616 & $1.67 \mathrm{E}-03$ \\
687 & 0.601232 & 1.202464 & 1.503080 & $1.52 \mathrm{E}-03$ \\
742 & 0.600980 & 1.201959 & 1.502449 & $1.47 \mathrm{E}-03$ \\
808 & 0.600492 & 1.200984 & 1.501230 & $1.20 \mathrm{E}-03$ \\
919 & 0.599984 & 1.199969 & 1.499961 & $1.07 \mathrm{E}-03$ \\
1,000 & 0.599685 & 1.199370 & 1.499213 & \\
\hline
\end{tabular}

Example 4.2 Take $u=(4,8,10)^{\top}, x^{0}=(1.2,1.2,1.2)^{\top}$, and

$$
\begin{aligned}
& C^{1}=\left\{x=\left(x_{1}, x_{2}, x_{3}\right)^{\top}: x_{1}^{2}+x_{2}^{2}+x_{3}^{2}-4 \leq 0\right\} \\
& C^{2}=\left\{x=\left(x_{1}, x_{2}, x_{3}\right)^{\top}:\left(x_{1}-1\right)^{2}+x_{2}^{2}+x_{3}^{2}-4 \leq 0\right\} .
\end{aligned}
$$

Use Algorithm 3.5 to calculate the projection $P_{C^{1} \cap C^{2}} u$.

Numerical results on Example 4.1 and Example 4.2 are provided in Table 1 and Table 2, respectively, as follows.

Competing interests

The authors declare that they have no competing interests.

Authors' contributions

All authors contributed equally to the writing of this paper. All authors read and approved the final manuscript.

Acknowledgements

This work was supported by National Natural Science Foundation of China (Grant No. 11201476) and the Fundamental Research Funds for the Central Universities (3122014K010).

Received: 21 August 2013 Accepted: 30 July 2014 Published: 21 Aug 2014

\section{References}

1. Yang, Q: On variable-set relaxed projection algorithm for variational inequalities. J. Math. Anal. Appl. 302, 166-179 (2005) 
2. Censor, Y, Elfving, T: A multiprojection algorithm using Bregman projection in product space. Numer. Algorithms 8(2-4), 221-239 (1994)

3. Yang, Q: The relaxed CQ algorithm for solving the split feasibility problem. Inverse Probl. 20, 1261-1266 (2004)

4. $\mathrm{Xu}, \mathrm{HK}$ : Iterative methods for the split feasibility problem in infinite-dimensional Hilbert spaces. Inverse Probl. 26 105018 (2010)

5. Zhang, W, Han, D, Li, Z: A self-adaptive projection method for solving the multiple-sets split feasibility problem. Inverse Probl. 25, 115001 (2009)

6. Zhao, J, Yang, Q: Self-adaptive projection methods for the multiple-sets split feasibility problem. Inverse Probl. 27 , 035009 (2011)

7. Von Neumann, J: In: Functional Operators, Vol. Il: The Geometry of Orthogonal Spaces. Annals of Mathematical Studies, vol. 22. Princeton University Press, Princeton (1950)

8. Bregman, LM: The method of successive projections for finding a common point of convex sets. Sov. Math. Dokl. 6 , 688-692 (1965)

9. Gubin, LG, Polyak, BT, Raik, EV: The method of projections for finding the common point of convex sets. USSR Comput. Math. Math. Phys. 7, 1-24 (1967)

10. Bruck, RE, Reich, S: Nonexpansive projections and resolvents of accretive operators in Banach spaces. Houst. J. Math. 3, 459-470 (1977)

11. Gibali, A, Censor, Y, Reich, S: The subgradient extragradient method for solving variational inequalities in Hilbert space. J. Optim. Theory Appl. 148, 318-335 (2011)

12. Hundal, HS: An alternating projection that does not converge in norm. Nonlinear Anal. 57, 35-61 (2004)

13. Fukushima, M: A relaxed projection method for variational inequalities. Math. Program. 35, 58-70 (1986)

14. López, G, Martín-Márquez, V, Wang, FH, Xu, HK: Solving the split feasibility problem without prior knowledge of matrix norms. Inverse Probl. (2012). doi:10.1088/0266-5611/28/8/085004

15. He, S, Yang, C: Solving the variational inequality problem defined on intersection of finite level sets. Abstr. Appl. Anal. (2013). doi:10.1155/2013/942315

16. Bauschke, HH, Borwein, JM: On projection algorithms for solving convex feasibility problem. SIAM Rev. 38, 367-426 (1996)

10.1186/1029-242X-2014-307

Cite this article as: He et al.: A simple algorithm for computing projection onto intersection of finite level sets. Journal of Inequalities and Applications 2014, 2014:307

\section{Submit your manuscript to a SpringerOpen ${ }^{\circ}$ journal and benefit from:}

- Convenient online submission

Rigorous peer review

- Immediate publication on acceptance

- Open access: articles freely available online

- High visibility within the field

- Retaining the copyright to your article 